\title{
Valuation of the Prepayment Option of a Perpetual Corporate Loan
}

\author{
Timothee Papin $^{1}$ and Gabriel Turinici ${ }^{2}$ \\ ${ }^{1}$ BNP Paribas CIB Resource Portfolio Management and CEREMADE, Université Paris Dauphine, 75016 Paris, France \\ ${ }^{2}$ CEREMADE, Université Paris Dauphine, 75016 Paris, France \\ Correspondence should be addressed to Gabriel Turinici; gabriel.turinici@dauphine.fr
}

Received 13 December 2012; Accepted 2 February 2013

Academic Editor: Dragoş-Pătru Covei

Copyright @ 2013 T. Papin and G. Turinici. This is an open access article distributed under the Creative Commons Attribution License, which permits unrestricted use, distribution, and reproduction in any medium, provided the original work is properly cited.

We investigate in this paper a perpetual prepayment option related to a corporate loan. The default intensity of the firm is supposed to follow a CIR process. We assume that the contractual margin of the loan is defined by the credit quality of the borrower and the liquidity cost that reflects the funding cost of the bank. Two frameworks are discussed: firstly a loan margin without liquidity cost and secondly a multiregime framework with a liquidity cost dependent on the regime. The prepayment option needs specific attention as the payoff itself is an implicit function of the parameters of the problem and of the dynamics. In the unique regime case, we establish quasianalytic formulas for the payoff of the option; in both cases we give a verification result that allows for the computation of the price of the option. Numerical results that implement the findings are also presented and are completely consistent with the theory; it is seen that when liquidity parameters are very different (i.e., when a liquidity crisis occurs) in the high liquidity cost regime, the exercise domain may entirely disappear, meaning that it is not optimal for the borrower to prepay during such a liquidity crisis. The method allows for quantification and interpretation of these findings.

\section{Introduction}

When a firm needs money, it can turn to its bank which lends it against, for example, periodic payments in a form of a loan. In almost every loan contract, the borrower has the option to prepay a portion or all the nominal at any time without penalties.

We assume in this model that the riskless interest rate, denoted by $r$, is constant and known. The liquidity cost dynamics will be described later. The interest rate of the loan is the sum of the constant interest rate, a margin defined according to the credit quality of the borrower, and a liquidity cost that reflects the funding costs of the lender, the bank.

In order to decide whether the exercise of the option is worthwhile, the borrower (the firm) compares the actualized value of the remaining payments with the nominal value to pay. If the remaining payments exceed the nominal value, then it is optimal for the borrower to refinance his debt at a lower rate.

When the borrower is subject to default, the computation of the actualization is less straightforward. It starts with considering all possible scenarios of evolution for the default intensity in a risk-neutral framework and computing the average value of the remaining payments (including the final payment of the principal if applicable); this quantity will be called " $P V R P$ " (denoted by $\xi$ ) and is the present value of the remaining payments, that is, the cash amount equivalent, both for borrower and lender in this model of the set of remaining payments. The PVRP is compared with the nominal: if the PVRP value is larger than the nominal, then the borrower should prepay, otherwise not. Recall that at the initial time the payments correspond to a rate, the sum of the interest rate and a contractual margin $\overline{\rho_{0}}$, which is precisely making the two quantities equal. Note that in order to compute the price of the embedded prepayment option, the lender also uses the PVRP as it will be seen below.

For a bank, the prepayment option is essentially a reinvestment risk, that is, the risk that the borrower decides to repay earlier his/her loan and that the bank cannot reinvest its excess of cash in a new loan. So the longer the maturity of the loan, the riskier the prepayment option. Therefore, it is interesting to study long-term loans that are set for 
more than three years and can run for more than twenty years. The valuation problem of the prepayment option can be modeled as an American embedded option on a risky debt owned by the borrower. As Monte-Carlo simulations are slow to converge and the binomial tree techniques are time consuming for long-term loans (cf. works by Cossin and $\mathrm{Lu}$ [1]), we decided to focus, in this paper, on the prepayment option for perpetual loan.

When valuing financial products with long maturity, the robustness with respect to shocks and other exogenous variabilities is important. Among problems that have to be treated is the liquidity and its variability. Liquidity is crucial for the stability of the financial system. Past events like the Asian crisis of 1997 [2]; the Russian financial crisis of 1998 [3]; the defaults of hedge funds and investment firms like LTCM, Enron, Worldcom, and Lehman Brothers defaults, sovereign debts crisis of 2010-11, and so on prove that banks hold significant liquidity risk in their balance sheets. A liquidity crisis can have a severe impact on bank's funding costs, its market access (reputation risk), and short-term funding capabilities.

Following the state of the economic environment, the liquidity can be defined by distinct states. Between two crises, investors are confident and banks find it easier to launch their long-term refinancing programs through regular bonds issuances. Thus the liquidity market is stable. Unfortunately, during crisis, liquidity becomes scarce, pushing the liquidity curve to very high levels which can only decrease if confidence returns to the market. The transition between these two distinct behaviors is rarely smooth but rather sudden.

In order to model the presence of distinct liquidity behaviors, we will simulate the liquidity cost by a continuous time Markov chain that can have a discrete set of possible values, one for each regime that is encountered in the liquidity evolution.

From a technical point of view, this paper faces several nonstandard conditions: although the goal is to value a perpetual American option, the payoff of the option is highly nonstandard (is dependent on the PVRP). As a consequence, the characterization of the exercise region is not standard and technical conditions have to be met. Furthermore, our focus here is on a specific type of dynamics (of CIR type) with even more specific interest on the situation when several regimes are present.

The balance of the paper is as follows: in the remainder of this section (Section 1.1) we review the related existing literature; in Section 2, we consider that the liquidity cost is negligible and that the borrower credit risk is defined by his/her default intensity (called in the following simply "intensity") which follows a CIR stochastic process. In this situation, we are able to obtain a quasianalytic formula for the PVRP. In Section 3 we explore the situation when the liquidity cost, defined as the cost of the lender to access the cash on the market, has several distinct regimes that we model by a Markov chain. We write the pricing formulas and theoretically support an algorithm to identify the boundary of the exercise region; numerical examples and concluding remarks close the paper.
1.1. Related Literature. There exist few articles (e.g., works by Cossin and $\mathrm{Lu}[1]$ ) on the loan prepayment option but a close subject, the prepayment option in a fixed-rate mortgage loan, has been widely covered in several papers by Hilliard et al. [4] and more recent works by Chen et al. [5]. To approximate the PDE satisfied by the prepayment option, they define two state variables (interest rate and house price). Their approach is based on a bivariate binomial option pricing technique with a stochastic interest rate and a stochastic house value.

Another contribution by Cossin and Lu [1] applies the binomial tree technique (but of course it is time consuming for long-term loans due to the nature of binomial trees) to corporate loans. They consider a prepayment option with a 1-year loan with a quarterly step, but it is difficult to have an accurate assessment of the option price for a 10-year loan.

There also exist mortgage prepayment decision models based on Poisson regression approach for mortgage loans (see, e.g., Schwartz and Torous [6]). Unfortunately, the volume and history of data are very weak in the corporate loan market.

Due to the form of their approach, these papers did not have to consider the geometry of the exercise region because it is explicitly given by the numerical algorithm. This is not the case for us and requires that particular care be taken when stating the optimality of the solution. Furthermore, to the best of our knowledge, none of these approaches explored the circumstance when several regimes exist.

The analysis of Markov-modulated regimes has been investigated in the literature when the underlying(s) follow the Black-Scholes dynamics with drift and volatility having Markov jumps; several works are of interest in this area: Guo and Zhang [7] have derived the closed-form solutions for vanilla American put; Guo analyses in [8] Russian (i.e., perpetual look-back) options and is able to derive explicit solutions for the optimal stopping time; in [9] $\mathrm{Xu}$ and $\mathrm{Wu}$ analyse the situation of a two-asset perpetual American option where the payoff function is a homogeneous function of degree one; Mamon and Rodrigo [10] find explicit solutions to vanilla European options. Buffington and Elliott [11] study European and American options and obtain equations for the price. A distinct approach (Wiener-Hopf factorization) is used by Jobert and Rogers [12] to derive very good approximations of the option prices for, among others, American puts. Other contributions include [13, 14].

Works involving Markov switched regimes and CIR dynamics appear in [15] where the bond valuation problem is considered (but not in the form of an American option; their approach will be relevant to the computation of the payoff of our American option although in their model only the mean reverting level is subject to Markov jumps) and in [16] where the term structure of the interest rates is analysed.

On the other hand numerical methods are proposed in [17] where it is found that a fixed point policy iteration coupled with a direct control formulation seems to perform best.

Finally, we refer to [18] for theoretical results concerning the pricing of American options in general. 


\section{Perpetual Prepayment Option with a Stochastic Intensity CIR Model}

We assume throughout the paper that the interest rate $r$ is constant. Therefore, the price of the prepayment option only depends on the intensity evolution over time. We model the intensity dynamics by a Cox-Ingersoll-Ross process (see [1921] for theoretical and numerical aspects of CIR processes and the situations where the CIR process has been used in finance):

$$
d \lambda_{s}=\gamma\left(\theta-\lambda_{s}\right) d s+\sigma \sqrt{\lambda_{s}} d W_{s}, \quad \gamma, \theta, \sigma>0, \quad \lambda_{0}=\overline{\lambda_{0}} .
$$

It is known that if $2 \gamma \theta \geq \sigma^{2}$, then CIR process ensures an intensity strictly positive. Fortunately, as it will be seen in the following, the PVRP is given by an analytic formula.

2.1. Analytical Formulas for the PVRP. Assume a loan with a fixed coupon defined by the interest rate $r$ and an initial contractual margin $\overline{\rho_{0}}$. Here $\overline{\rho_{0}}$ does not take into account any commercial margin (see Remark 1 ). Let $\xi(t, T, \lambda)$ be the present value of the remaining payments at time $t$ of a corporate loan with initial contractual margin $\overline{\rho_{0}}$ (depending on $\overline{\lambda_{0}}$ ), intensity at time $t, \lambda_{t}$, following the risk-neutral equation (1) with $\lambda_{t}=\lambda$; the loan has nominal amount $K$ and contractual maturity $T$. Here the assignment $\lambda_{t}=\lambda$ means that the dynamics of $\lambda_{t}$ start at time $t$ from the numerical value $\lambda$. All random variables will be conditional by this event (see, e.g., (3)).

Therefore the loan value $\operatorname{LV}(t, T, \lambda)$ is equal to the present value of the remaining payments $\xi(t, T, \lambda)$ minus the prepayment option value $P(t, T, \lambda)$ :

$$
\operatorname{LV}(t, T, \lambda)=\xi(t, T, \lambda)-P(t, T, \lambda) .
$$

The present value of the cash flows discounted at the (instantaneous) risky rate $r+\lambda_{t}$ is denoted by $\xi$. The infinitesimal cash flow at time $t$ is $K\left(r+\overline{\rho_{0}}\right)$ and the final payment of the principal $K$. Then

$$
\begin{aligned}
& \xi(t, T, \lambda) \\
& \quad=\mathbb{E}\left[K \cdot\left(r+\overline{\rho_{0}}\right) \int_{t}^{T} e^{-\int_{t}^{\tilde{t}}\left(r+\lambda_{u}\right) d u} d \widetilde{t}+K e^{-\int_{t}^{T} r+\lambda_{u} d u} \mid \lambda_{t}=\lambda\right] .
\end{aligned}
$$

For a perpetual loan the maturity $T=+\infty$. Since $\lambda_{t}$ is always positive, $r+\lambda_{t}>0$, and thus the last term tends to zero when $T \rightarrow \infty$. A second remark is that since $\gamma, \theta$, and $\sigma$ are independent of time, $\xi$ is independent of the starting time $t$ :

$$
\begin{aligned}
\xi(t, \lambda) & =\mathbb{E}\left[K \cdot\left(r+\overline{\rho_{0}}\right) \int_{t}^{+\infty} e^{-\int_{t}^{\tilde{t}} r+\lambda_{u} d u} d \tilde{t} \mid \lambda_{t}=\lambda\right] \\
& =\mathbb{E}\left[K \cdot\left(r+\overline{\rho_{0}}\right) \int_{0}^{+\infty} e^{-\int_{0}^{\tilde{t}} r+\lambda_{u} d u} d \tilde{t} \mid \lambda_{0}=\lambda\right] \\
& =: \xi(\lambda),
\end{aligned}
$$

where the last equality is a definition. For a CIR stochastic process, we obtain (see $[19,21])$

$$
\xi(\lambda)=K \cdot\left(r+\overline{\rho_{0}}\right) \int_{0}^{+\infty} e^{-r \tilde{t}} B(0, \tilde{t}, \lambda) d \widetilde{t}
$$

where for general $t, \tilde{t}$ we use the notation

$$
B(t, \tilde{t}, \lambda)=\mathbb{E}\left[e^{-\int_{t}^{\tau} \lambda_{u} d u} \mid \lambda_{t}=\lambda\right] .
$$

Note that $B(t, \tilde{t}, \lambda)$ is a familiar quantity, and analytic formulas are available for (7) (see Lando [22, page 292]). The intensity is following a CIR dynamic; therefore, for general $t, \tilde{t}$

$$
B(t, \widetilde{t}, \lambda)=\alpha(t, \widetilde{t}) e^{-\beta(t, \widetilde{t}) \lambda}
$$

with

$$
\begin{gathered}
\alpha(t, \tilde{t})=\left(\frac{2 h e^{(\gamma+h)((\widetilde{t}-t) / 2)}}{2 h+(\gamma+h)\left(e^{(\tilde{t}-t) h}-1\right)}\right)^{2 \gamma \theta / \sigma^{2}}, \\
\beta(t, \tilde{t})=\frac{2\left(e^{(\tilde{t}-t) h}-1\right)}{2 h+(\gamma+h)\left(e^{(\widetilde{t}-t) h}-1\right)}, \\
\text { where } h=\sqrt{\gamma^{2}+2 \sigma^{2}},
\end{gathered}
$$

where $\gamma$ and $\sigma$ are the parameters of the CIR process of the intensity in (1). Obviously $B(0, t, \lambda)$ is monotonic with respect to $\lambda$; thus the same holds for $\xi$.

The margin $\overline{\rho_{0}}$ is the solution of the following equilibrium equation:

$$
\xi\left(\overline{\lambda_{0}}\right)=K
$$

which can be interpreted as the fact that the present value of the cash flows (according to the probability of survival) is equal to the nominal $K$ :

$$
\overline{\rho_{0}}=\frac{1}{\int_{0}^{+\infty} e^{-r \tilde{t}} B\left(0, \widetilde{t}, \overline{\lambda_{0}}\right) d \widetilde{t}}-r .
$$

Note that we assume no additional commercial margin.

Remark 1. If an additional commercial margin $\mu_{0}$ is considered, then $\overline{\rho_{0}}$ is first computed as above and then replaced by $\overline{\overline{\rho_{0}}}=\overline{\rho_{0}}+\mu_{0}$ in (6). Equations (10) and (11) will not be verified as such but will still hold with some $\overline{\overline{\lambda_{0}}}$ instead of $\overline{\lambda_{0}}$; for instance, we will have

$$
\overline{\overline{\rho_{0}}}=\frac{1}{\int_{0}^{+\infty} e^{-r \tilde{t}} B\left(0, \tilde{t}, \overline{\overline{\lambda_{0}}}\right) d \widetilde{t}}-r .
$$

With these changes all results in the paper are valid, except that when computing for operational purposes once the price of the prepayment option is computed for all $\lambda$, one will use $\lambda=\overline{\lambda_{0}}$ as price relevant to practice. 
Remark 2. Some banks allow (per year) a certain percentage of the prepaid amount without penalty and the rest with a penalty. This circumstance could be incorporated into the model by changing the definition of the payoff by subtracting the penalty. This will impact formula (15). 1 ; thus

From definition (7) of $B(t, \tilde{t}, \lambda)$ it follows that $B(t, \tilde{t}, \lambda)<$

$$
e^{-r \tilde{t}} B\left(0, \tilde{t}, \overline{\lambda_{0}}\right)<e^{-r \tilde{t}}
$$

and as a consequence

$$
\int_{0}^{+\infty} e^{-r \tilde{t}} B\left(0, \tilde{t}, \overline{\lambda_{0}}\right) d \widetilde{t}<\int_{0}^{+\infty} e^{-r \tilde{t}} d \widetilde{t}=\frac{1}{r}
$$

which implies that $\overline{\rho_{0}}>0$.

2.2. Valuation of the Prepayment Option. The valuation problem of the prepayment option can be modeled as an American call option on a risky debt owned by the borrower. Here the prepayment option allows borrower to buy back and refinance his/her debt according to the current contractual margin at any time during the life of the option. As the perpetual loan, the option value will be assumed independent of the time $t$.

As discussed above, the prepayment exercise results in a payoff $(\xi(t, T, \lambda)-K)^{+}$for the borrower. The option is therefore an American call option on the risky asset $\xi\left(t, T, \lambda_{t}\right)$ and the principal $K$ (the amount to be reimbursed) being the strike. Otherwise we can see it as an American option on the risky $\lambda_{t}$ with payoff

$$
\chi(t, \lambda):=(\xi(t, \lambda)-K)^{+}
$$

or, for our perpetual option,

$$
\chi(\lambda):=(\xi(\lambda)-K)^{+} .
$$

We will denote by $\mathscr{A}$ the characteristic operator (cf. [23, Chapter 7.5]) of the CIR process, that is, the operator that acts on any $C^{2}$ class function $v$ by

$$
(\mathscr{A} v)(\lambda)=\gamma(\theta-\lambda) \partial_{\lambda} v(\lambda)+\frac{1}{2} \sigma^{2} \lambda \partial_{\lambda \lambda} v(\lambda)
$$

Denote for $a, b \in \mathbb{R}$ and $x \geq 0$ by $U(a, b, x)$ the solution to the confluent hypergeometric differential (also known as the Kummer) equation [24]:

$$
x z^{\prime \prime}(x)+(b-x) z^{\prime}(x)-a z(x)=0
$$

that increases at most polynomially at infinity and is finite (not null) at the origin. Recall also that this function is proportional to the confluent hypergeometric function of the second kind $U(a, b, x)$ (also known as the Kummer function of the second kind, Tricomi function, or Gordon function); for $a, x>0$ the function $U(a, b, x)$ is given by the formula

$$
U(a, b, x)=\frac{1}{\Gamma(a)} \int_{0}^{+\infty} e^{-x t} t^{a-1}(1+t)^{b-a-1} d t
$$

When $a \leq 0$, one uses other representations (see the cited references; for instance, one can use a direct computation or the recurrence formula $U(a, b, x)=(2 a-b+z-2) U(a+$ $1, b, x)-(a+1)(a-b+2) U(a+2, b, x))$ it is known that $U(a, b, x)$ behaves as $x^{-a}$ at infinity. Also introduce for $x \geq 0$

$$
\begin{aligned}
W(x)= & e^{x\left((\gamma-h) / \sigma^{2}\right)} x^{\left(\sigma^{2}-2 \gamma \theta\right) / \sigma^{2}} \\
& \times U\left(-\frac{-r \sigma^{2}-\sigma^{2} h+\gamma^{2} \theta+\gamma h \theta}{\sigma^{2} h}, 2-\frac{2 \gamma \theta}{\sigma^{2}}, \frac{2 h}{\sigma^{2}} x\right),
\end{aligned}
$$

where $h=\sqrt{\gamma^{2}+2 \sigma^{2}}$.

Theorem 3. (1) Introduce for $\Lambda>0$ the family of functions: $P_{\Lambda}(\lambda)$ such that

$$
\begin{gathered}
P_{\Lambda}(\lambda)=\chi(\lambda) \quad \forall \lambda \in[0, \Lambda], \\
\left(\mathscr{A} P_{\Lambda}\right)(\lambda)-(r+\lambda) P_{\Lambda}(\lambda)=0, \quad \forall \lambda>\Lambda, \\
\lim _{\lambda \rightarrow \Lambda} P_{\Lambda}(\lambda)=\chi(\Lambda), \\
\lim _{\lambda \rightarrow \infty} P_{\Lambda}(\lambda)=0 .
\end{gathered}
$$

Then

$$
P_{\Lambda}(\lambda)= \begin{cases}\chi(\lambda) & \forall \lambda \in[0, \Lambda] \\ \frac{\chi(\Lambda)}{W(\Lambda)} W(\lambda) & \forall \lambda \geq \Lambda .\end{cases}
$$

(2) Suppose now a $\left.\Lambda^{*} \in\right] 0, \overline{\rho_{0}} \wedge \overline{\lambda_{0}}$ [ exists such that

$$
\left.\frac{d P_{\Lambda^{*}}(\lambda)}{d \lambda}\right|_{\lambda=\left(\Lambda^{*}\right)^{+}}=\left.\frac{d \chi(\lambda)}{d \lambda}\right|_{\lambda=\left(\Lambda^{*}\right)^{-}} .
$$

Then the price of the prepayment option is $P(\lambda)=P_{\Lambda^{*}}(\lambda)$.

Proof. We start with the first item: it is possible to obtain a general solution of (22) in an analytic form. We recall that $z(X)=U(a, b, X)$ is the solution of the Kummer equation (18). A cumbersome but straightforward computation shows that the general solution vanishing at infinity of the PDE (22) is $W(\lambda)$; thus

$$
P_{\Lambda}(\lambda)=C_{\Lambda} W(\lambda) \quad \forall \lambda>\Lambda
$$

with some $C_{\Lambda}>0$ to be determined. Now use the boundary conditions. If $\lambda=\Lambda$, by continuity $\chi(\Lambda)=P_{\Lambda}(\Lambda)=C_{\Lambda} W(\Lambda)$. Thus, $C_{\Lambda}=\chi(\Lambda) / W(\Lambda)$. Division by $W$ is legitimate because by definition, $W(x)>0$ for all $x>0$.

We now continue with the second part of the theorem. The valuation problem of an American option goes through several steps: first one introduces the admissible trading and consumption strategies (cf. [25, Chapter 5]); then one realizes using results in the cited reference (also see $[21,26]$ ) that the price $P(\lambda)$ of the prepayment option involves computing a stopping time associated to the payoff. Denote by $\mathscr{T}$ the ensemble of (positive) stopping times; we conclude that

$$
P(\lambda)=\sup _{\tau \in \mathscr{T}} \mathbb{E}\left(e^{-\int_{0}^{\tau} r+\lambda_{u} d u} \chi\left(\lambda_{\tau}\right) \mid \lambda_{0}=\lambda\right) .
$$


Further results derived for the situation of a perpetual (standard) American put options [18, 27] show that the stopping time has a simple structure: a critical level exists that splits the positive axis into two regions: the exercise region (to the left) where it is optimal to exercise and where the price equals the payoff and a continuation region (to the right) where the price satisfies a partial differential equation similar to Black-Scholes equation. We refer to [28] for how to adapt the theoretical arguments for the situation when the dynamics is not Black-Scholes-like but a CIR process.

The result builds heavily on the fact that the discounted payoff of the standard situation of an American put $e^{-r t}(S-$ $K)^{-}$is a submartingale. For us the discounted payoff is

$$
e^{-\int_{0}^{t} r+\lambda_{u} d u} \chi\left(\lambda_{t}\right)=e^{-\int_{0}^{t} r+\lambda_{u} d u}\left(\xi\left(\lambda_{t}\right)-K\right)^{+},
$$

and checking this condition requires here more careful examination which is the object of Lemma 4. It is now possible to apply Theorem 10.4.1 [23, Section 10.4, page 227] (see also [28] for specific treatment of the CIR process) which will show that $P(\lambda)$ is the true option price if the following conditions are satisfied:

(1) on $] 0, \Lambda^{*}$ [ we have $P(\lambda)=\chi(\lambda)=(\xi(\lambda)-K)^{+}$and the relation (35) holds;

(2) the solution candidate $P(\lambda)$ satisfies the relation

$$
(\mathscr{A} P)(\lambda)-(r+\lambda) P(\lambda)=0, \quad \forall \lambda>\Lambda^{*} ;
$$

(3) the function $P(\lambda)$ is $C^{1}$ everywhere, continuous at the origin and $C^{2}$ on each subinterval $] 0, \Lambda^{*}[$ and ]$\Lambda^{*}, \infty[$.

The theorem also says that the borrower exercises his option on the exercise region $\left[0, \Lambda^{*}\right]$ while on the continuation region $] \Lambda^{*}, \infty[$ the borrower keeps the option because it is worth more nonexercised.

We now show that $P_{\Lambda^{*}}$ verifies all conditions above which will allow to conclude that $P=P_{\Lambda^{*}}$. The requirement 1 is proved in Lemma 4; the requirement 3 amounts to asking that the optimal frontier value $\Lambda^{*}$ is chosen such that

$$
\left.\frac{d P_{\Lambda^{*}}(\lambda)}{d \lambda}\right|_{\lambda=\left(\Lambda^{*}\right)^{+}}=\left.\frac{d \chi(\lambda)}{d \lambda}\right|_{\lambda=\left(\Lambda^{*}\right)^{-}} .
$$

The requirement 2 implies that in the continuation region the price is the solution of the following PDE:

$$
(\mathscr{A} P)(\lambda)-(r+\lambda) P(\lambda)=0, \quad \forall \lambda>\Lambda^{*} .
$$

For this PDE we need boundary conditions. The condition at $\lambda=\Lambda^{*}$ is

$$
\left.P(\lambda)\right|_{\lambda=\Lambda^{*}}=\left.\chi(\lambda)\right|_{\lambda=\Lambda^{*}}
$$

When $\lambda=+\infty$, the default intensity is infinite; thus the time to failure is zero, and thus the borrower has failed; in this case the option is worthless; that is,

$$
\lim _{\lambda \rightarrow \infty} P(\lambda)=0 .
$$

These conditions give exactly the definition of $P_{\Lambda^{*}}$.
Lemma 4. The following inequality holds:

$$
(\mathscr{A} \chi)(\lambda)-(r+\lambda) \chi(\lambda)<0, \quad \forall \lambda<\overline{\rho_{0}} \wedge \overline{\lambda_{0}} .
$$

Proof. Recall that $\chi(\lambda)=(\xi(\lambda)-K)^{+}$; definition (5) of $\xi$ implies (cf. [23, Section 8.2 and exercise 9.12, page 203]) that $\xi$ is solution of the following PDE:

$$
(\mathscr{A} \xi)(\lambda)-(r+\lambda) \xi(\lambda)+\left(r+\overline{\rho_{0}}\right) K=0, \quad \forall \lambda>0 .
$$

For $\lambda<\overline{\lambda_{0}}$ we have $\xi(\lambda)>K=\xi\left(\overline{\lambda_{0}}\right)$; thus

$$
\begin{aligned}
\left(\mathscr{A}(\xi(\cdot)-K)^{+}\right)(\lambda)-(r+\lambda)(\xi(\lambda)-K)^{+} \\
\quad=(\mathscr{A}(\xi(\cdot)-K))(\lambda)-(r+\lambda)(\xi(\lambda)-K) \\
\quad=(\mathscr{A} \xi)(\lambda)-(r+\lambda) \xi(\lambda)+(r+\lambda) K \\
\quad=-\left(r+\overline{\rho_{0}}\right) K+(r+\lambda) K \\
\quad=\left(\lambda-\overline{\rho_{0}}\right) K<0 \quad \forall \lambda<\overline{\rho_{0}} \wedge \overline{\lambda_{0}} .
\end{aligned}
$$

Note that Theorem 3 is only a sufficient result (a socalled "verification" result); under the assumption that a $\Lambda^{*}$ fulfilling the hypotheses of the theorem exists the question is how to find it.

Two approaches can be considered; first, it is enough to find a zero of the following function $\Lambda \mapsto \Upsilon(\Lambda)$ := $\left(\left.\left(\partial P_{\Lambda}(\lambda) / \partial \lambda\right)\right|_{\lambda=\Lambda^{+}}-\left.(\partial \chi(\lambda) / \partial \lambda)\right|_{\lambda=\Lambda^{-}}\right)$(the last equality is a definition). Of course $\left.(\partial \chi(\lambda) / \partial \lambda)\right|_{\lambda=\overline{\lambda_{0}}+\epsilon}=0$ and $\left.\left(\partial P_{\overline{\lambda_{0}}+\epsilon}(\lambda) / \partial \lambda\right)\right|_{\lambda=\overline{\lambda_{0}}+\epsilon}<0$; thus $\Upsilon\left(\overline{\lambda_{0}}+\epsilon\right)<0$ for any $\epsilon>0$ and hence $\Upsilon\left(\overline{\lambda_{0}}\right) \leq 0$. Thus it is natural not to look for $\Lambda^{*}$ outside the interval $\left[0, \overline{\lambda_{0}}\right]$. The theorem asks furthermore to restrict the search to the interval $\left[0, \overline{\lambda_{0}} \wedge \overline{\rho_{0}}\right]$.

A different convenient procedure to find the critical $\Lambda^{*}$ is to consider the dependence $\Lambda \mapsto P_{\Lambda}\left(\overline{\lambda_{0}}\right)$. Let us consider the stopping time $\tau_{\Lambda}$ that stops upon entering the domain $[0, \Lambda]$. We remark that by a Feynman-Kac formula (cf. [23, page 203]),

$$
P_{\Lambda}(\lambda)=\mathbb{E}\left(e^{-\int_{0}^{\tau_{\Lambda}} r+\lambda_{u} d u} \chi\left(\lambda_{\tau_{\Lambda}}\right) \mid \lambda_{0}=\lambda\right) .
$$

From (28) $P(\lambda) \geq P_{\Lambda}(\lambda)$ for any $\lambda$; thus $\Lambda^{*}$ is the value that maximizes (with respect to $\Lambda$ ) the function $\Lambda \mapsto P_{\Lambda}\left(\overline{\lambda_{0}}\right)$. To comply with the theorem, the maximization is performed in the interval $\left[0, \overline{\lambda_{0}} \wedge \overline{\rho_{0}}\right]$.

2.3. Numerical Application. We consider a perpetual loan $(T=+\infty)$ with a nominal amount $K=1$ and the borrower default intensity $\lambda_{t}$ follows a CIR dynamics with parameters: initial intensity $\overline{\lambda_{0}}=300 \mathrm{bps}$, volatility $\sigma=0.05$, average intensity $\theta=200 \mathrm{bps}$, and reversion coefficient $\gamma=0.5$. We assume a constant interest rate $r=300 \mathrm{bps}$; that is, $r=3 \%$. Recall that a basis point, denoted by "1 bps", equals $10^{-4}$.

In order to find the initial contractual margin, we use (11) and find $\overline{\rho_{0}}=208$ bps. 


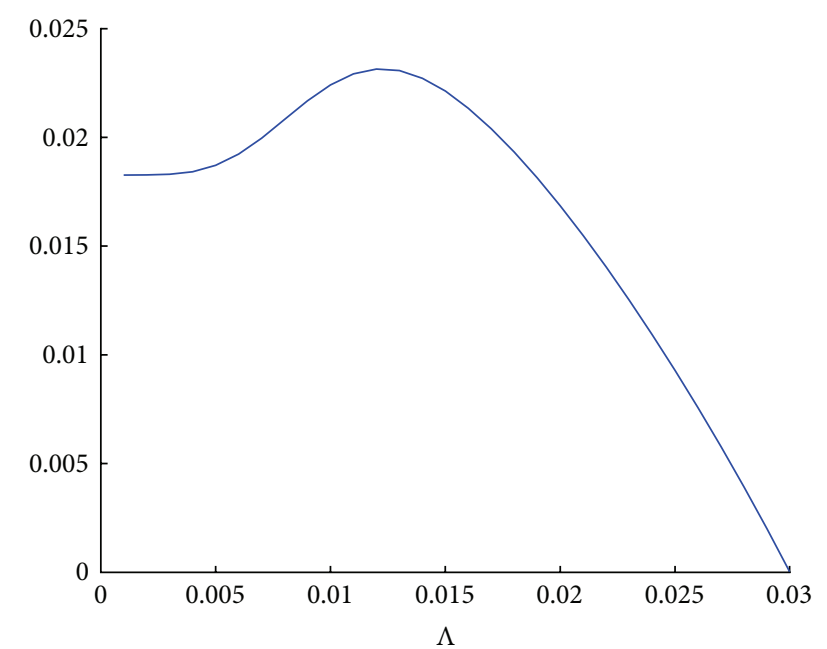

FIGURE 1: We illustrate here the dependence of $P_{\Lambda}\left(\overline{\lambda_{0}}\right)$ as a function of $\Lambda$; this allows for finding the optimal value $\Lambda^{*}$ that maximizes the option price. For the numerical example described here we obtain $\Lambda^{*}=123$ bps.

At inception, the present value of cash flows is at par, so $\xi\left(\overline{\lambda_{0}}\right)=1$. The prepayment option price is $P\left(+\infty, \overline{\lambda_{0}}\right)=$ 0.0232 ; that is, $P\left(\overline{\lambda_{0}}\right)=2.32 \% \cdot K$. Therefore the loan value equals $\xi\left(\overline{\lambda_{0}}\right)-P\left(\overline{\lambda_{0}}\right)=0,9768$.

The value $\Lambda^{*}=123$ bps is obtained by maximizing $P_{\Lambda}\left(\overline{\lambda_{0}}\right)$ as indicated in the remarks above; the dependence of $P_{\Lambda}\left(\overline{\lambda_{0}}\right)$ with respect to $\Lambda$ is illustrated in Figure 1. The loan value will be equal to par if the intensity decreases until the exercise region $\left(\lambda<\Lambda^{*}\right)$ (see Figure 2$)$. The continuation and exercise regions are depicted in Figure 3. We postpone to Section 3.5 the description of the numerical method to solve (22).

\section{Perpetual Prepayment Option with a Switching Regime}

In this second part, the perpetual prepayment option is still an option on the credit risk, intensity, and also the liquidity cost. The liquidity cost is defined as the specific cost of a bank to access the cash on the market. This cost will be modeled with a switching regime with a Markov chain of finite states of the economy. The interest rate $r$ is still assumed constant. Therefore, the assessment of the loan value and its prepayment option is an $\mathrm{N}$-dimensional problem. The intensity is still defined by a Cox-Ingersoll-Ross process with $2 k \theta \geq \sigma^{2}$ :

$$
d \lambda_{t}=\gamma\left(\theta-\lambda_{t}\right) d t+\sigma \sqrt{\lambda_{t}} d W_{t}, \quad \lambda_{0}=\overline{\lambda_{0}}
$$

3.1. Theoretical Regime Switching Framework. We assume the economic state of the market is described by a finite state Markov chain $\mathbf{X}=\left\{X_{t}, t \geq 0\right\}$. The state space $\mathbf{X}$ can be taken to be, without loss of generality, the set of unit vectors $E=\left\{e_{1}, e_{2}, \ldots, e_{N}\right\}, e_{i}=(0, \ldots, 0,1,0, \ldots, 0)^{T} \in \mathbb{R}^{N}$. Here $T$ is the transposition operator.

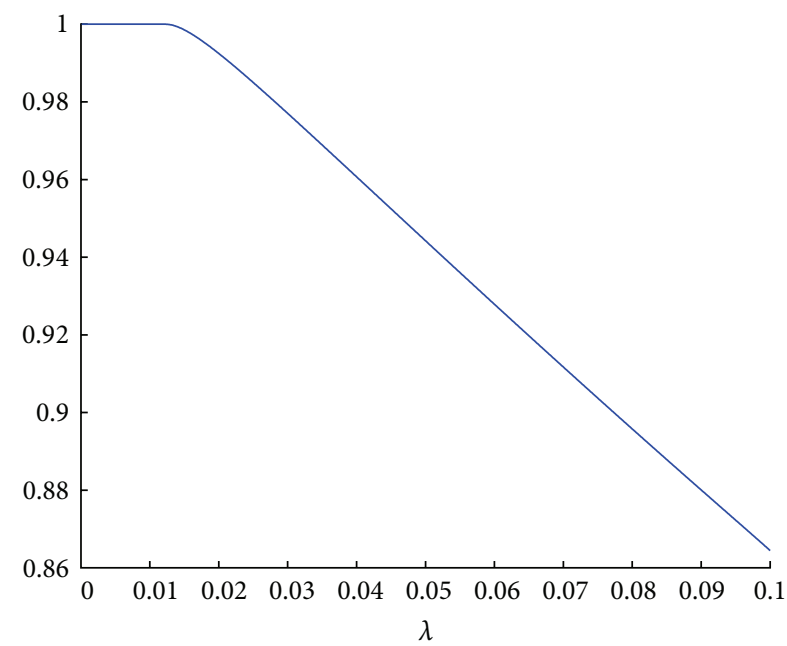

FIGURE 2: Loan value as a function of the intensity. The loan value is decreasing when there is a degradation of the credit quality (i.e., $\lambda$ increases) and converges to 0 .

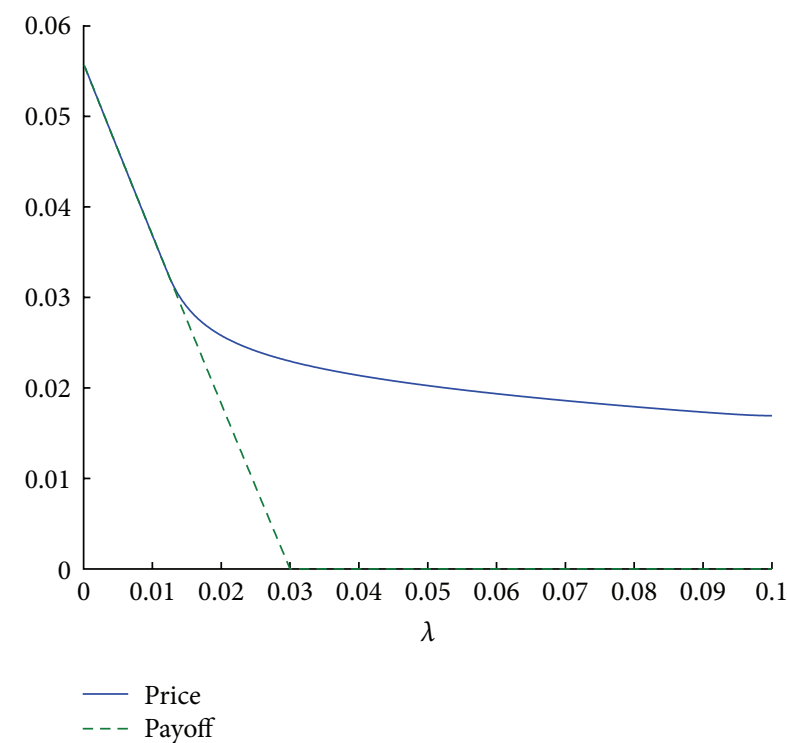

FIGURE 3: Prepayment option price $P(\lambda)$ (solid line) and payoff $\chi(\lambda)$ (dashed line) as a function of the intensity $\lambda$. Two regions appear: the continuation region $\lambda>\Lambda^{*}$ and the exercise region $\lambda \leq \Lambda^{*}$.

Assuming the process $X_{t}$ is homogeneous in time and has a rate matrix $A$, then if $p_{t}=\mathbb{E}\left[X_{t}\right] \in \mathbb{R}^{N}$,

$$
\begin{gathered}
\frac{d p_{t}}{d t}=A p_{t} \\
X_{t}=X_{0}+\int_{0}^{t} A X_{u} d u+M_{t},
\end{gathered}
$$

where $\mathbf{M}=\left\{M_{t}, t \geq 0\right\}$ is a martingale with respect to the filtration generated by $\mathbf{X}$. In differential form

$$
d X_{t}=A X_{t} d t+d M_{t}, \quad X_{0}=\overline{X_{0}} .
$$


We assume the instantaneous liquidity cost of the bank depends on the state $\mathbf{X}$ of the economy, so that

$$
l_{t}=\left\langle\mathbf{1}, X_{t}\right\rangle \text {. }
$$

Denote by $a_{k, j}$ the entry on the line $k$ and the column $j$ of the $N \times N$ matrix $A$ with $a_{k, j} \geq 0$ for $j \neq k$ and $\sum_{j=1}^{N} a_{k, j}=0$ for any $k$.

3.2. Analytical Formulas for the PVRP. Assume a loan has a fixed coupon defined by the constant interest rate $r$ and an initial contractual margin $\overline{\rho_{0}}$ calculated at the inception for a par value of the loan. Let $\xi\left(t, T, \lambda_{t}, X_{t}\right)$ be the present value of the remaining payments at time $t$ of a corporate loan, where $\lambda_{t}$ is the intensity at time $t, T$ is the contractual maturity, $K$ is the nominal amount, and $X_{t}$ is the state of the economy at time $t$.

The loan value $\operatorname{LV}(t, T, \lambda)$ is still equal to the present value of the remaining payments $\xi(t, T, \lambda)$ minus the prepayment option value $P(t, T, \lambda)$ :

$$
\mathrm{LV}(t, T, \lambda)=\xi(t, T, \lambda)-P(t, T, \lambda) .
$$

The PVRP $\xi$ is the present value of the cash flows discounted at the risky rate, where the risky rate at time $t$ is the constant risk-free rate $r$ plus the liquidity $\cos t l_{t}$ and the intensity $\lambda_{t}$. Similar to the discussion in the Section $2.1, \xi$ is not depending on time when $T=+\infty$ (perpetual loan). So we denote that,

$$
\begin{aligned}
\xi(\lambda, X):= & K\left(r+\overline{\rho_{0}}\right) \\
& \times \mathbb{E}\left[\int_{0}^{+\infty} e^{-\int_{0}^{\tilde{t}} r+l_{u}+\lambda_{u} d u} d \tilde{t} \mid \lambda_{0}=\lambda, X_{0}=X\right] .
\end{aligned}
$$

We consider that there is no correlation between the credit risk, that is, the intensity $\lambda_{t}$, of the borrower and the cost to access the cash on the market, that is, the liquidity cost $l_{t}$, of the lender. Therefore, we have

$$
\begin{aligned}
\xi(\lambda, X)=K\left(r+\overline{\rho_{0}}\right) \int_{0}^{+\infty} & e^{-r \tilde{t}} \mathbb{E}\left[e^{-\int_{0}^{\tilde{t}} \lambda_{u} d u} \mid \lambda_{0}=\lambda\right] \\
& \times \mathbb{E}\left[e^{-\int_{0}^{\tilde{\tau}} l_{u} d u} \mid X_{0}=X\right] d \tilde{t} .
\end{aligned}
$$

Remark 5. The crucial information here is that the coefficients $\gamma, \theta$, and $\sigma$ of the CIR process are not depending on the regime $X$; thus we can separate the CIR dynamics and the Markov dynamics at this level. A different approach can extend this result by using the properties of the PVRP as explained in the next section.

Note that (cf. Section 2.1, equation (7))

$$
\mathbb{E}\left[e^{-\int_{0}^{t} \lambda_{u} d u} \mid \lambda_{0}=\lambda\right]=B(0, t, \lambda)
$$

and $B(0, t, \lambda)$ is evaluated using (8)-(11). In order to compute

$$
\mathbb{E}\left[e^{-\int_{0}^{\tilde{t}} l_{u} d u} \mid X_{0}=X\right]
$$

let $f_{k}(t)$ be defined by

$$
f_{k}(t)=\mathbb{E}\left[e^{-\int_{0}^{t} l_{u} d u} \mid X_{0}=e_{k}\right] .
$$

Let $\tau$ be the time of the first jump from $X_{0}=\left\langle\mathbf{X}, e_{k}\right\rangle$ to some other state. We know (cf. Lando [22] paragraph 7.7, page 211) that $\tau$ is a random variable following an exponential distribution of parameter $\alpha_{k}$ with

$$
\alpha_{k}=\sum_{j \neq k} a_{k, j}
$$

We also know that conditional to the fact that a jump has occurred at time $\tau$, the probability that the jump is from state $e_{k}$ to state $e_{j}$ is $p_{k, j}$, where

$$
p_{k, j}=\frac{a_{k, j}}{\alpha_{k}} .
$$

Thus,

$$
\begin{aligned}
f_{k}(t)= & \mathbb{P}(\tau>t) e^{-l_{k} t} \\
& +\mathbb{P}(\tau \leq t) e^{-l_{k} \tau} \\
& \times \sum_{j \neq k} \mathbb{P}\left(l_{\tau}=l_{j}\right) \mathbb{E}\left[e^{-\int_{\tau}^{t} l_{u} d u} \mid X_{\tau}=\left\langle\mathbf{X}, e_{j}\right\rangle\right] \\
= & e^{-\left(l_{k}+\alpha_{k}\right) t}+\alpha_{k} \int_{0}^{t} e^{-\left(l_{k}+\alpha_{k}\right) \tau} \sum_{j \neq k} p_{k, j} f_{j}(t-\tau) d \tau .
\end{aligned}
$$

Then,

$$
\begin{aligned}
e^{\left(l_{k}+\alpha_{k}\right) t} f_{k}(t) & =1+\alpha_{k} \int_{0}^{t} e^{\left(l_{k}+\alpha_{k}\right)(t-\tau)} \sum_{j \neq k} p_{k, j} f_{j}(t-\tau) d \tau \\
& =1+\alpha_{k} \int_{0}^{t} e^{\left(l_{k}+\alpha_{k}\right) s} \sum_{j \neq k} p_{k, j} f_{j}(s) d s .
\end{aligned}
$$

By differentiation with respect to $t$,

$$
\frac{d}{d t}\left[e^{\left(l_{k}+\alpha_{k}\right) t} f_{k}(t)\right]=\alpha_{k} e^{\left(l_{k}+\alpha_{k}\right) t} \sum_{j \neq k} p_{k, j} f_{j}(t) .
$$

Then

$$
\frac{d f_{k}(t)}{d t}+\left(l_{k}+\alpha_{k}\right) f_{k}(t)=\alpha_{k} \sum_{j \neq k} p_{k, j} f_{j}(t) .
$$

Thus,

$$
\frac{d f_{k}(t)}{d t}=\left[\sum_{j \neq k} \alpha_{k} p_{k, j} f_{j}(t)\right]-\left(l_{k}+\alpha_{k}\right) f_{k}(t) .
$$

Denote by $F(t)$ the vector $\left(f_{1}(t), f_{2}(t), \ldots, f_{N}(t)\right)^{T}$ and introduce the $N \times N$ matrix $B$

$$
B_{i, j}= \begin{cases}\alpha_{i} p_{i, j} & \text { if } i \neq j \\ -\left(\alpha_{i}+l_{i}\right) & \text { if } i=j .\end{cases}
$$


From (55) we obtain

$$
\frac{d F(t)}{d t}=B F(t) \quad \text { thus } F(t)=e^{B t} F(0)
$$

with the initial condition

$$
F(0)=\left(f_{k}(0)\right)_{k=1}^{N}=(1,1, \ldots, 1)^{T} \in \mathbb{R}^{N} .
$$

We have therefore analytic formulas for the $\operatorname{PVRP} \xi(\lambda, X)$. We refer the reader to [15] for similar considerations on related CIR switched dynamics.

Remark 6. When all liquidity parameters $l_{k}$ are equal (to some quantity $l$ ), then $B=A-l \cdot I d$, and then we obtain (after some computations) that $f_{k}(t)=e^{-l t}$; thus the payoff is equal to that of one-regime dynamics with interest rate $r+l$, which is consistent with intuitive image we may have. Another limiting case is when the switching is very fast (see also Remark 10 item 5 for further details).

The margin $\overline{\rho_{0}}$ is set to satisfy the equilibrium equation:

$$
\xi\left(\overline{\lambda_{0}}, \overline{X_{0}}\right)=K
$$

Similar arguments to that in previous section show that $\overline{\rho_{0}}>$ $\min _{k} l_{k}>0$. See Remark 1 for the situation when an additional commercial margin is to be considered.

We will also need to introduce for any $k=1, \ldots, N$ the value $\bar{\Lambda}_{k}^{0}$ such that

$$
\xi\left(\bar{\Lambda}_{k}^{0}, e_{k}\right)=K
$$

Of course, $\bar{\Lambda}_{\bar{X}_{0}}^{0}=\overline{\lambda_{0}}$. Recall that $\xi\left(\lambda, e_{k}\right)$ is decreasing with respect to $\lambda$; when $\xi\left(0, e_{k}\right)<K$, there is no solution to (59) and we will choose by convention $\bar{\Lambda}_{k}^{0}=0$.

3.3. Further Properties of the PVRP $\xi$. It is useful for the following to introduce a PDE formulation for $\xi$. To ease the notations, we introduce the operator $\mathscr{A}^{\mathscr{R}}$ that acts on functions $v(\lambda, X)$ as follows:

$$
\begin{aligned}
\left(\mathscr{A}^{\mathscr{R}} v\right)\left(\lambda, e_{k}\right)= & (\mathscr{A} v)\left(\lambda, e_{k}\right)-\left(r+l_{k}+\lambda\right) v\left(\lambda, e_{k}\right) \\
& +\sum_{j=1}^{N} a_{k, j}\left(v\left(\lambda, e_{j}\right)-v\left(\lambda, e_{k}\right)\right) .
\end{aligned}
$$

Having defined the dynamics (39) and (41), one can use an adapted version of the Feynman-Kac formula in order to conclude that PVRP defined by (44) satisfies the equation

$$
\left(\mathscr{A}^{\mathscr{R}} \xi\right)+\left(r+\overline{\rho_{0}}\right) K=0 .
$$

Remark 7. When the dynamics involve different coefficients of the CIR process for different regimes (cf. also Remark 5), (62) changes in that it will involve, for $\xi\left(\cdot, e_{k}\right)$, the operator

$$
\mathscr{A}_{k}(v)(\lambda)=\gamma_{k}\left(\theta_{k}-\lambda\right) \partial_{\lambda} v(\lambda)+\frac{1}{2} \sigma_{k}^{2} \lambda \partial_{\lambda \lambda} v(\lambda)
$$

instead of $\mathscr{A}$.
3.4. Valuation of the Prepayment Option. The valuation problem of the prepayment option can be modeled as an American call option on a risky debt owned by the borrower with payoff:

$$
\chi(\lambda, X)=(\xi(\lambda, X)-K)^{+} .
$$

Here the prepayment option allows borrower to buy back and refinance his/her debt according to the current contractual margin at any time during the life of the option.

Theorem 8. For any $N$-tuple $\Lambda=\left(\Lambda_{k}\right)_{k=1}^{N} \in\left(\mathbb{R}_{+}\right)^{N}$ introduce the function $P_{\Lambda}(\lambda, X)$ such that

$$
\begin{gathered}
P_{\Lambda}\left(\lambda, e_{k}\right)=\chi\left(\lambda, e_{k}\right) \quad \forall \lambda \in\left[0, \Lambda_{k}\right], \\
\left(\mathscr{A}^{\mathscr{R}} P_{\Lambda}\right)\left(\lambda, e_{k}\right)=0, \quad \forall \lambda>\Lambda_{k}, k=1, \ldots, N, \\
\lim _{\lambda \rightarrow \Lambda_{k}} P_{\Lambda}\left(\lambda, e_{k}\right)=\chi\left(\Lambda_{k}, e_{k}\right), \quad \text { if } \Lambda_{k}>0, k=1, \ldots, N,
\end{gathered}
$$

$$
\lim _{\lambda \rightarrow \infty} P_{\Lambda}\left(\lambda, e_{k}\right)=0, \quad k=1, \ldots, N
$$

Suppose a $\Lambda^{*} \in \prod_{k=1}^{N}\left[0,\left(\overline{\rho_{0}}-l_{k}\right)^{+} \wedge \bar{\Lambda}_{k}^{0}\right]$ exists such that for all $k=1, \ldots, N$ :

$$
\begin{gathered}
P_{\Lambda^{*}}(\lambda, X) \geq \chi(\lambda, X) \quad \forall \lambda, X, \\
\left.\frac{\partial P_{\Lambda^{*}}\left(\lambda, e_{k}\right)}{\partial \lambda}\right|_{\lambda=\left(\Lambda_{k}^{*}\right)^{+}}=\left.\frac{\partial \chi\left(\lambda, e_{k}\right)}{\partial \lambda}\right|_{\lambda=\left(\Lambda_{k}^{*}\right)^{-}} \quad \text { if } \Lambda_{k}^{*}>0,
\end{gathered}
$$

$$
\begin{array}{r}
\sum_{j=1}^{N} a_{k, j}\left(P_{\Lambda^{*}}\left(\lambda, e_{j}\right)-\chi\left(\lambda, e_{j}\right)\right)+K\left(\lambda+l_{k}-\overline{\rho_{0}}\right) \leq 0 \\
\forall \lambda \in \min _{j} \Lambda_{j}^{*}, \Lambda_{k}^{*}[
\end{array}
$$

Then $P=P_{\Lambda^{*}}$.

Proof. Similar arguments as in the proof of Theorem 3 lead to consider the American option price in the form

$$
P(\lambda, X)=\sup _{\tau \in \mathscr{T}} \mathbb{E}\left[e^{-\int_{0}^{\tau} r+l_{u}+\lambda_{u} d u} \chi\left(\lambda_{\tau}, X_{\tau}\right) \mid \lambda_{0}=\lambda, X_{0}=X\right] .
$$

We note that for $\Lambda \in\left(\mathbb{R}_{+}^{*}\right)^{N}$ if $\tau_{\Lambda}$ is the stopping time that stops upon exiting the domain $\lambda>\Lambda_{k}$ when $X=e_{k}$, then

$$
\begin{aligned}
& P_{\Lambda}(\lambda, X) \\
& \quad=\mathbb{E}\left[e^{-\int_{0}^{\tau_{\Lambda}} r+l_{u}+\lambda_{u} d u} \chi\left(\lambda_{\tau_{\Lambda}}, X_{\tau_{\Lambda}}\right) \mid \lambda_{0}=\lambda, X_{0}=X\right] .
\end{aligned}
$$

Remark that for $\Lambda \in\left(\mathbb{R}_{+}^{*}\right)^{N}$ the stopping time $\tau_{\Lambda}$ is finite a.e. Thus for any $\Lambda \in\left(\mathbb{R}_{+}^{*}\right)^{N}$ we have $P \geq P_{\Lambda}$; when $\Lambda$ has some null coordinates, the continuity (ensured among others by the boundary condition (65)) shows that we still have $P \geq P_{\Lambda}$. In 
particular for $\Lambda^{*}$ we obtain $P \geq P_{\Lambda^{*}}$; all that remains to be proved is the reverse inequality; that is, $P \leq P_{\Lambda^{*}}$.

To this end we use a similar technique as in Theorem 10.4.1 [23, Section 10.4, page 227] (see also [7] for similar considerations). First one can invoke the same arguments as in the cited reference (cf. Appendix D for technicalities) and work as if $P_{\Lambda^{*}}$ is $C^{2}$ (not only $C^{1}$ as the hypothesis ensures).

Denote by $D_{\Lambda^{*}}$ the set $\left\{\left(\lambda, e_{k}\right) \mid \lambda \in\left[0, \Lambda_{k}^{*}\right], k=\right.$ $1, \ldots, N\}$ (which will be the exercise region), and denote by $C_{\Lambda^{*}}$ its complementary with respect to $\mathbb{R}_{+} \times E$ (which will be the continuation region).

Lemma 9 shows that $\mathscr{A}^{\mathscr{R}} P_{\Lambda^{*}}$ is nonpositive everywhere (and is null on $C_{\Lambda^{*}}$ ). The Itô formula shows that

$$
\begin{aligned}
& d\left(e^{-\int_{0}^{t} r+l_{s}+\lambda_{s} d s} P_{\Lambda^{*}}\left(\lambda_{t}, X_{t}\right)\right) \\
& \quad=e^{-\int_{0}^{t} r+l_{s}+\lambda_{s} d s}\left(\mathscr{A}^{\mathscr{R}} P_{\Lambda^{*}}\right)\left(\lambda_{t}, X_{t}\right) d t+d(\text { martingale }) .
\end{aligned}
$$

Taking averages and integrating from 0 to some stopping time $\tau$, it follows from $\mathscr{A}^{\mathscr{R}} P_{\Lambda^{*}} \leq 0$ that

$$
\begin{aligned}
P_{\Lambda^{*}}(\lambda, X) & \geq \mathbb{E}\left[e^{-\int_{0}^{\tau} r+l_{u}+\lambda_{u} d u} P_{\Lambda^{*}}\left(\lambda_{\tau}, X_{\tau}\right) \mid \lambda_{0}=\lambda, X_{0}=X\right] \\
& \geq \mathbb{E}\left[e^{-\int_{0}^{\tau} r+l_{u}+\lambda_{u} d u} \chi\left(\lambda_{\tau}, X_{\tau}\right) \mid \lambda_{0}=\lambda, X_{0}=X\right] .
\end{aligned}
$$

Since this is true for any stopping time $\tau$, the conclusion follows.

Lemma 9. Under the hypothesis of the Theorem 8 the following inequality holds (strongly except for the values $(\lambda, X)=$ $\left(\Lambda_{j}^{*}, e_{k}\right)$ and everywhere in a weak sense):

$$
\left(\mathscr{A}^{\mathscr{R}} P_{\Lambda^{*}}\right)(\lambda, X) \leq 0, \quad \forall \lambda>0, \forall X
$$

Proof. The nontrivial part of this lemma comes from the fact that if for fixed $k$ we have for $\lambda$ in a neighborhood of some $\lambda_{1}: P_{\Lambda^{*}}\left(\lambda, e_{k}\right)=\chi\left(\lambda, e_{k}\right)$, this does not necessarily imply $\left(\mathscr{A}^{\mathscr{R}} P_{\Lambda^{*}}\right)\left(\lambda_{1}, e_{k}\right)=\left(\mathscr{A}^{\mathscr{R}} \chi\right)\left(\lambda_{1}, e_{k}\right)$ because $\mathscr{A}^{\mathscr{R}}$ depends on other values $P_{\Lambda^{*}}\left(\lambda, e_{j}\right)$ with $j \neq k$.

From (66) the conclusion is trivially verified for $X=e_{k}$ for any $\lambda \in] \Lambda_{k}^{*}, \infty[$.

We now analyse the situation when $\lambda<\min _{j} \Lambda_{j}^{*}$; this means in particular that $0 \leq \lambda<\min _{j} \Lambda_{j}^{*} \leq \bar{\Lambda}_{\ell}^{0}$ for any $\ell$; thus $\bar{\Lambda}_{\ell}^{0}>0$. Note that $\Lambda_{k}^{*}<\bar{\Lambda}_{k}^{0}$ implies $\xi\left(\Lambda_{k}^{*}, e_{k}\right) \geq \xi\left(\bar{\Lambda}_{k}^{0}, e_{k}\right)=K$ for any $k=1, \ldots, N$; thus $\chi\left(\lambda, e_{k}\right)=\xi\left(\lambda, e_{k}\right)-K$ for any $\lambda \in\left[0, \Lambda_{k}^{*}\right]$ and any $k$. Furthermore since $\lambda<\min _{j} \Lambda_{j}^{*}$, we have $P_{\Lambda^{*}}\left(\lambda, e_{k}\right)=\chi\left(\lambda, e_{k}\right)=\xi\left(\lambda, e_{k}\right)-K$ for any $k$. Fix $X=e_{k}$; then

$$
\begin{aligned}
\left(\mathscr{A}^{\mathscr{R}} P_{\Lambda^{*}}\right)\left(\lambda, e_{k}\right) & =\left(\mathscr{A}^{\mathscr{R}} \chi\right)\left(\lambda, e_{k}\right)=\left(\mathscr{A}^{\mathscr{R}}(\xi-K)\right)\left(\lambda, e_{k}\right) \\
& =\left(\mathscr{A}^{\mathscr{R}} \xi\right)\left(\lambda, e_{k}\right)-\mathscr{A}^{\mathscr{R}}(K) \\
& =-\left(r+\overline{\rho_{0}}\right) K-\left(r+l_{k}+\lambda\right) K \\
& =K\left(l_{k}+\lambda-\overline{\rho_{0}}\right) \\
& \leq K\left(l_{k}+\Lambda_{k}^{*}-\overline{\rho_{0}}\right) \leq 0,
\end{aligned}
$$

the last inequality being true by hypothesis.

A last situation is when $\lambda \in] \min _{j} \Lambda_{j}^{*}, \Lambda_{k}^{*}[$; then $P_{\Lambda^{*}}\left(\lambda, e_{k}\right)=\chi\left(\lambda, e_{k}\right)$ but some terms $P_{\Lambda^{*}}\left(\lambda, e_{j}\right)$ for $j \neq k$ may differ from $\chi\left(\lambda, e_{j}\right)$. More involved arguments are invoked in this case. This point is specific to the fact that the payoff $\chi$ itself has a complex structure and as such was not emphasized in previous works (e.g., [7], etc.).

Recalling the properties of $\xi$, one obtains (and since $\left.P_{\Lambda^{*}}\left(\lambda, e_{k}\right)=\chi\left(\lambda, e_{k}\right)\right)$ the following:

$$
\begin{aligned}
\left(\mathscr{A}^{\mathscr{R}} P_{\Lambda^{*}}\right)\left(\lambda, e_{k}\right)= & (\mathscr{A} \chi)\left(\lambda, e_{k}\right)-\left(r+l_{k}+\lambda\right) \chi\left(\lambda, e_{k}\right) \\
& +\sum_{j=1}^{N} a_{k, j}\left(P_{\Lambda^{*}}\left(\lambda, e_{j}\right)-\chi\left(\lambda, e_{k}\right)\right) \\
= & \left(\mathscr{A}^{\mathscr{R}} \chi\right)\left(\lambda, e_{k}\right) \\
& +\sum_{j=1}^{N} a_{k, j}\left(P_{\Lambda^{*}}\left(\lambda, e_{j}\right)-\chi\left(\lambda, e_{j}\right)\right) \\
= & \left(\mathscr{A}^{\mathscr{R}} \xi\right)\left(\lambda, e_{k}\right)-\mathscr{A}^{\mathscr{R}}(K) \\
& +\sum_{j=1}^{N} a_{k, j}\left(P_{\Lambda^{*}}\left(\lambda, e_{j}\right)-\chi\left(\lambda, e_{j}\right)\right) \\
= & -K\left(r+\overline{\rho_{0}}\right)+\left(r+l_{k}+\lambda\right) K \\
& +\sum_{j=1}^{N} a_{k, j}\left(P_{\Lambda^{*}}\left(\lambda, e_{j}\right)-\chi\left(\lambda, e_{j}\right)\right) \leq 0,
\end{aligned}
$$

where for the last inequality we use hypothesis (71). Finally, since we proved that $\left(\mathscr{A}^{\mathscr{R}} P_{\Lambda^{*}}\right)(\lambda, X) \leq 0$ strongly except for the values $(\lambda, X)=\left(\Lambda_{j}^{*}, e_{k}\right)$ and since $P_{\Lambda^{*}}$ is of $C^{1}$ class, we obtain the conclusion (the weak formulation only uses the first derivative of $P_{\Lambda^{*}}$ ).

Remark 10. Several remarks are in order at this point.

(1) When only one regime is present; that is, $N=1$, the hypothesis of the theorem is identical to that of Theorem 3 since (71) is automatically satisfied.

(2) When $N>1$ checking (71) does not involve any computation of derivatives and is straightforward. 
(3) As mentioned in the previous section, the theorem is a verification result, that is, it only gives sufficient conditions for a candidate to be the option price. Two possible partial converse results are possible: the first one to prove that the optimal price is indeed an element of the family $P_{\Lambda}$. The second converse result is to prove that supposing $P=P_{\Lambda^{*}}$, then $\Lambda^{*} \epsilon$ $\prod_{k=1}^{N}\left[0,\left(\overline{\rho_{0}}-l_{k}\right)^{+} \wedge \bar{\Lambda}_{k}^{0}\right]$, and (69)-(71) are satisfied.

(4) The search for the candidate $\Lambda^{*}$ can be done either by looking for a zero of the function $\Lambda \mapsto \Upsilon(\Lambda):=$ $\left(\left.\left(\partial P_{\Lambda^{*}}\left(\lambda, e_{k}\right) / \partial \lambda\right)\right|_{\lambda=\left(\Lambda_{k}^{*}\right)^{+}}-\left.\left(\partial \chi\left(\lambda, e_{k}\right) / \partial \lambda\right)\right|_{\lambda=\left(\Lambda_{k}^{*}\right)^{-}}\right)_{k=1}^{N}$ or by maximizing on $\left.\prod_{k=1}^{N}\right] 0,\left(\overline{\rho_{0}}-l_{k}\right) \wedge \bar{\Lambda}_{k}^{0}[$ the function $\Lambda \mapsto P_{\Lambda}\left(\overline{\lambda_{0}}, \overline{X_{0}}\right)$.

(5) If the optimization of $P_{\Lambda}\left(\overline{\lambda_{0}}, \overline{X_{0}}\right)$ is difficult to perform, one can use a continuation argument with respect to the coupling matrix $A$. Denote by $\Lambda^{*}(A)$ the optimal value of $\Lambda^{*}$ as function of $A$. When $A=$ 0 , each $\Lambda_{k}^{*}$ is found as in Section 2 (the problem separates into $N$ independent, that is, no coupled, valuation problems, each of which requiring to solve a one-dimensional optimization) and we construct thus $\Lambda^{*}(0)$. When considering $\mu A$ with $\mu \rightarrow \infty$ at the limit, the optimal $\Lambda_{*}(\infty A)$ has all entries equal to $\Lambda_{\text {mean }}^{*}$, where $\Lambda_{\text {mean }}^{*}$ is the optimal value for oneregime $(N=1)$ dynamics with riskless interest rate $r$ being replaced by $r+\left(\sum_{k=1}^{N} l_{k} / \alpha_{k}\right) /\left(\sum_{k=1}^{N} 1 / \alpha_{k}\right)$. Having established the two extremal points, the candidate $\Lambda^{*}(A)$ is searched within the $N$-dimensional segment $\left[\Lambda^{*}(0), \Lambda^{*}(\infty A)\right]$.

3.5. Numerical Application. The numerical solution of the partial differential equation (66) is required. We use a finite difference method. The first derivative is approximated by the finite difference formula

$$
\frac{\partial}{\partial \lambda} P_{\Lambda}(\lambda, X)=\frac{P_{\Lambda}(\lambda+\delta \lambda, X)-P_{\Lambda}(\lambda-\delta \lambda, X)}{2 \delta \lambda}+O\left(\delta \lambda^{2}\right),
$$

while the second derivative is approximated by

$$
\begin{aligned}
\frac{\partial^{2}}{\partial \lambda^{2}} P_{\Lambda}(\lambda, X) & \\
= & \frac{P_{\Lambda}(\lambda+\delta \lambda, X)-2 P_{\Lambda}(\lambda+\delta \lambda, X)+P_{\Lambda}(\lambda-\delta \lambda, X)}{\delta \lambda^{2}} \\
& +O\left(\delta \lambda^{2}\right) .
\end{aligned}
$$

To avoid working with an infinite domain, a well-known approach is to define an artificial boundary $\lambda_{\max }$. Then a boundary condition is imposed on $\lambda_{\max }$ which leads to a numerical problem in the finite domain $\cup_{k=1}^{N}\left[\Lambda_{k}^{*}, \lambda_{\max }\right]$. In this numerical application, $\lambda_{\max }=400 \mathrm{bps}$. We discretize $\left[\Lambda^{*}, \lambda_{\max }\right]$ with a grid such that $\delta \lambda=1$ bps. Two approaches have been considered for imposing a boundary value at $\lambda_{\text {max }}$ : either consider that $P_{\Lambda}\left(\lambda_{\max }, e_{k}\right)=0, \forall k=$

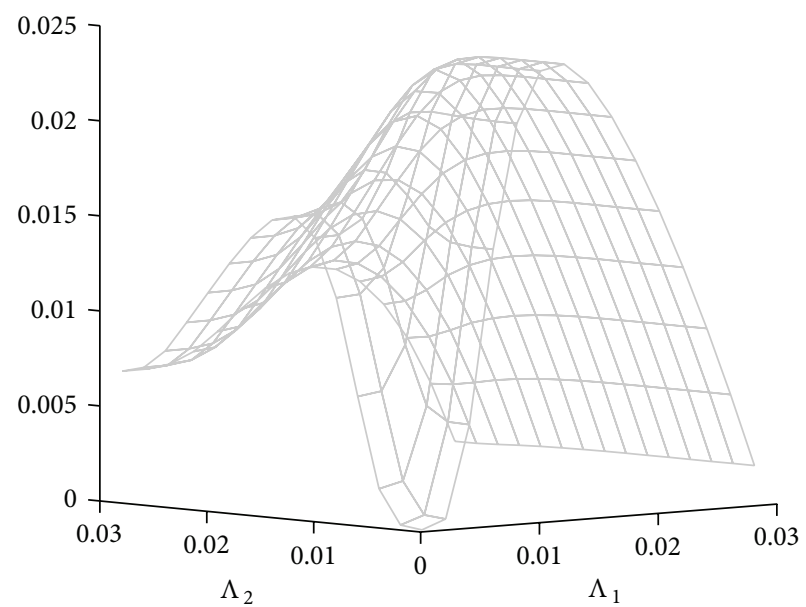

FIgURE 4: We illustrate here the dependence of $P_{\Lambda}\left(\overline{\lambda_{0}}, \overline{X_{0}}\right)$ as a function of $\Lambda$; this allows for finding the optimal $\left(\Lambda_{1}^{*}=122 \mathrm{bps}\right.$, $\Lambda_{2}^{*}=64 \mathrm{bps}$ ) that maximizes the option price.

$1, \ldots, N$ (homogeneous Dirichlet boundary condition) or that $(\partial / \partial \lambda) P_{\Lambda}\left(\lambda_{\max }, e_{k}\right)=0, \forall k=1, \ldots, N$ (homogeneous Neumann boundary condition). Both are correct in the limit $\lambda_{\max } \rightarrow \infty$. We tested the precision of the results by comparing with numerical results obtained on a much larger grid (10 times larger) while using the same $\delta \lambda$. The Neumann boundary condition gives much better results for the situations we considered and as such was always chosen (see also Figure 6).

We consider a perpetual loan with a nominal amount $K=$ 1 and the borrower default intensity $\lambda_{t}$ follows CIR dynamics with parameters: initial intensity $\overline{\lambda_{0}}=300 \mathrm{bps}$, volatility $\sigma=$ 0.05 , average intensity $\theta=200 \mathrm{bps}$, and reversion coefficient $\gamma=0.5$. We assume a constant interest rate $r=1 \%$ and a liquidity cost defined by a Markov chain of two states $l_{1}=$ $150 \mathrm{bps}$ and $l_{2}=200 \mathrm{bps}$. For $N=2$ the rate $A$ matrix is completely defined by $\alpha_{1}=1 / 3, \alpha_{2}=1$.

In order to find the initial contractual margin, we use (11) and find $\overline{\rho_{0}}=331 \mathrm{bps}$ in the state 1 . The contractual margin takes into account the credit risk (default intensity) and the liquidity cost. We have thus $\bar{\Lambda}_{1}^{0}=\overline{\lambda_{0}}$; we obtain then $\bar{\Lambda}_{2}^{0}=$ 260 bps.

The optimal value $\Lambda^{*}$ is obtained by maximizing $P_{\Lambda}\left(\overline{\lambda_{0}}, \overline{X_{0}}\right)$ and turns out to be $\left(\Lambda_{1}^{*}, \Lambda_{2}^{*}\right)=(122 \mathrm{bps}, 64 \mathrm{bps})$ (see Figure 4 ). To be accepted, this numerical solution has to verify all conditions of Theorem 8 . Hypotheses (69) and (71) are satisfied (see Figure 6) and hypothesis (71) is accepted after calculation. Moreover $\Lambda_{1}^{*} \leq\left(\overline{\rho_{0}}-l_{1}\right) \wedge \bar{\Lambda}_{1}^{0}$ and the analogous holds for $\Lambda_{2}^{*}$.

In the state $\overline{X_{0}}=1$, the present value of cash flows is at par, so $\xi\left(\overline{\lambda_{0}}, \overline{X_{0}}\right)=1$. The prepayment option price is $P\left(\overline{\lambda_{0}}, \overline{X_{0}}\right)=0.0240$. Therefore the loan value equals $\xi\left(\overline{\lambda_{0}}, \overline{X_{0}}\right)-P\left(\overline{\lambda_{0}}, \overline{X_{0}}\right)=0.9760$.

The loan value will be equal to the nominal if the intensity decreases until the exercise region $\lambda \leq \Lambda^{*}$ (see Figure 5). The continuation and exercise regions are depicted in Figure 6. 


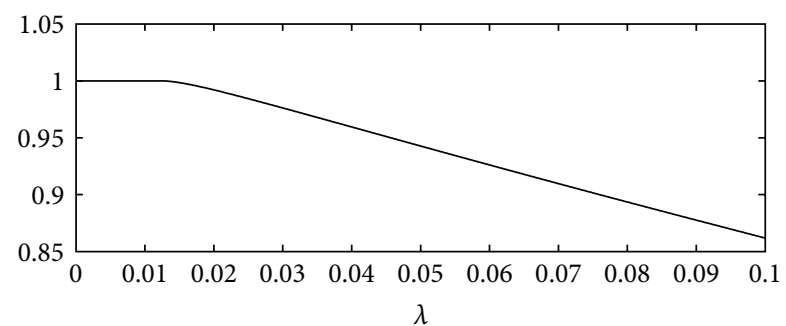

(a)

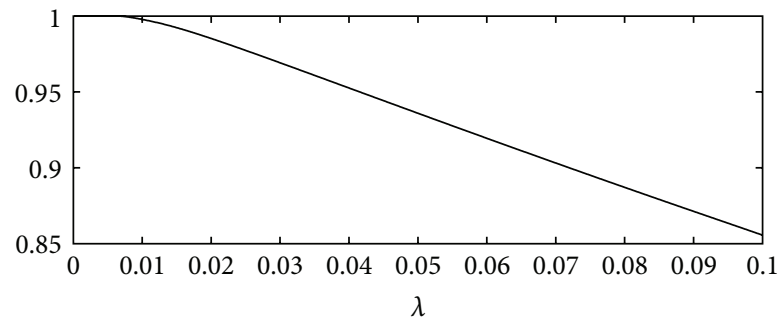

(b)

FIGURE 5: Loan value as a function of the intensity. (a) Regime $X=e_{1}$; (b) regime $X=e_{2}$. The loan value is decreasing when there is a degradation of the credit quality (i.e., when $\lambda$ increases) and converges to 0 .

3.6. Regimes in Which Is Never Optimal to Exercise. When the liquidity parameters corresponding to given regimes are very different, it may happen that the optimization of $P_{\Lambda}\left(\overline{\lambda_{0}}, \overline{X_{0}}\right)$ over $\Lambda$ gives an optimum value $\Lambda^{*}$ with some null coordinates $\Lambda_{k_{i}}, i=1, \ldots$. This may hint to the fact that in this situation it is never optimal to exercise during the regimes $e_{k_{i}}, i=1, \ldots$. This is not surprising in itself (remember that this is the case of an American call option) but needs more care when being dealt with. Of course when in addition $\bar{\Lambda}_{k_{i}}^{0}=0$, the payoff being null, it is intuitive that the option should not be exercised.

Remark 11. Further examination of Theorem 3 calls for the following remarks.

(1) The boundary value set in (65) for some regime $e_{k}$ with $\Lambda_{k}^{*}=0$ deserves an interpretation. The boundary value does not serve to enforce continuity of $\lambda \mapsto P_{\Lambda}(\lambda)$ because there is no exercise region in this regime. Moreover when $2 \gamma \theta \geq \sigma^{2}$, the intensity $\lambda_{u}$ does not touch 0 ; thus the stopping time $\tau_{\Lambda^{*}}$ is infinite in the regime $e_{k}$ (thus the boundary value in 0 is never used and thus need not be enforced); from a mathematical point of view it is known that no boundary conditions are required at points where the leading order differential operator is degenerate.

(2) It is interesting to know when such a situation can occur and how can one interpret it. Let us take a tworegime case $(N=2): l_{1}$ a "normal" regime and $l_{2}$ the "crisis" regime $\left(l_{2} \geq l_{1}\right)$; when the agent contemplates prepayment, the more severe the crisis (i.e., larger $\left.l_{2}-l_{1}\right)$, the less he/she is likely to prepay during the crisis when the cash is expensive (high liquidity

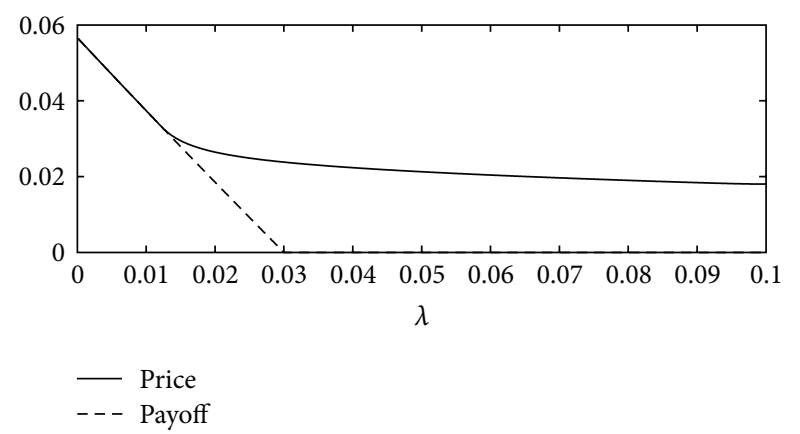

(a)

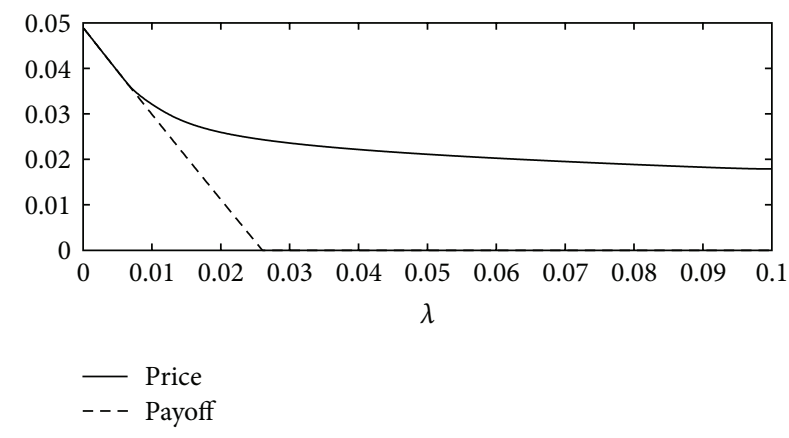

(b)

FIGURE 6: The price of the prepayment option $P_{\Lambda^{*}}(\lambda)$ (solid line) and the payoff $\chi(\lambda)$ (dashed line) as a function of the intensity $\lambda$. (a) Regime $X=e_{1}$; (b) regime $X=e_{2}$. For each regime two regions appear: the continuation region $\lambda>\Lambda_{i}^{*}$ and the exercise region $\lambda \leq$ $\Lambda_{i}^{*}$.

cost). We will most likely see that for $l_{1}=l_{2}$ some exercise regions exist while starting from some large $l_{2}$ the exercise region will disappear in regime $e_{2}$. This is completely consistent with the numerical results reported in this paper.

3.7. Numerical Application. We consider the same situation as in Section 3.7 except that $l_{1}=50 \mathrm{bps}$ and $l_{2}=250 \mathrm{bps}$. In order to find the initial contractual margin, we use (11) and find $\overline{\rho_{0}}=305 \mathrm{bps}$ in the state 1 . The contractual margin takes into account the credit risk (default intensity) and the liquidity cost. As before $\bar{\Lambda}_{1}^{0}=\overline{\lambda_{0}}$ but here we obtain $\bar{\Lambda}_{2}^{0}=$ 221 bps.

The couple $\left(\Lambda_{1}^{*}=121 \mathrm{bps}, \Lambda_{2}^{*}=0\right)$ (see Figure 7 ) maximizes $P_{\Lambda}\left(\overline{\lambda_{0}}, \overline{X_{0}}\right)$. There does not exist an exercise boundary in the state 2 . The loan value will equal the par if the intensity decreases until the exercise region $\lambda \leq \Lambda^{*}$ (see Figure 8 ). The continuation and exercise regions are depicted in Figure 9.

To be accepted as true price, the numerical solution $P_{\Lambda^{*}}$ has to verify all hypotheses and conditions of Theorem 8 . In the regime $X=e_{1}$, hypotheses (69) and (70) are verified numerically (see also Figure 9) and hypothesis (71) is accepted after calculation. Moreover $\Lambda_{k}^{*} \leq\left(\overline{\rho_{0}}-l_{k}\right) \wedge \bar{\Lambda}_{k}^{0}$ for $k=1,2$. 


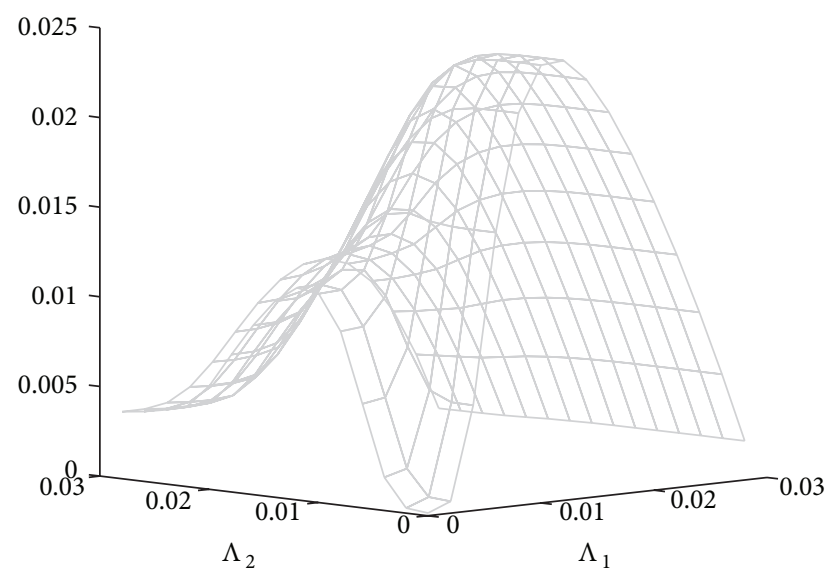

Figure 7: We illustrate here the dependence of $P_{\Lambda}\left(\overline{\lambda_{0}}, \overline{X_{0}}\right)$ as a function of the exercise boundary $\Lambda$; this allows to find the optimal $\left(\Lambda_{1}^{*}=121 \mathrm{bps}, \Lambda_{2}^{*}=0\right)$ that maximizes the option price.

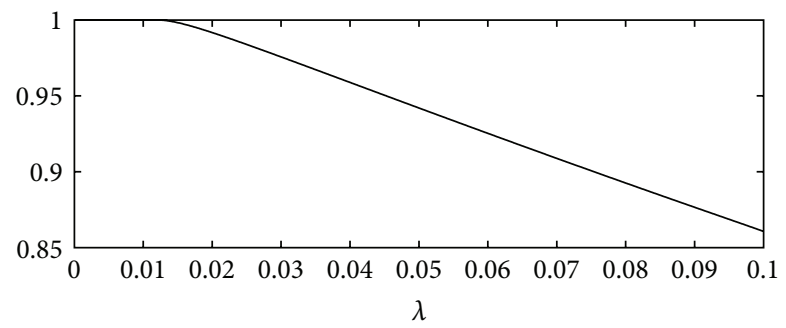

(a)

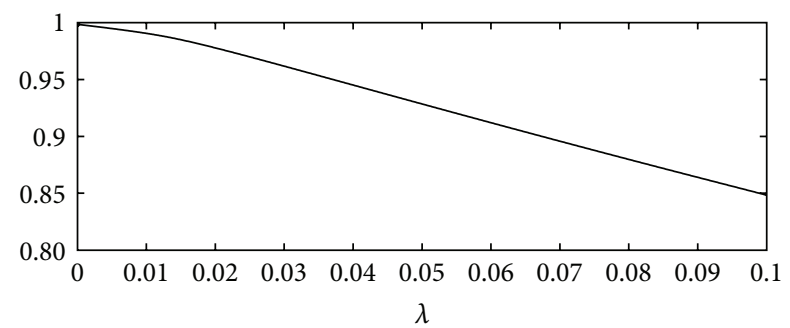

(b)

Figure 8: Loan value as a function of the intensity. (a) Regime $X=e_{1}$; (b) regime $X=e_{2}$. The loan value is decreasing when there is a degradation of the credit quality (i.e., when $\lambda$ increases) and converges to 0 .

In the state $X=e_{1}$, the present value of cash flows is at par, so $\xi\left(\overline{\lambda_{0}}, \overline{X_{0}}\right)=K=1$. The prepayment option price is $P\left(\overline{\lambda_{0}}\right)=0.0245$. Therefore the loan value LV equals $\xi\left(\overline{\lambda_{0}}\right)-$ $P\left(\overline{\lambda_{0}}\right)=0.9755$.

\section{Concluding Remarks}

We proved in this paper two sufficient theoretical results concerning the prepayment option of corporate loans. In our model the interest rate is constant, the default intensity follows a CIR process, and the liquidity cost follows a discrete space Markov jump process. The theoretical results were implemented numerically and show that the prepayment

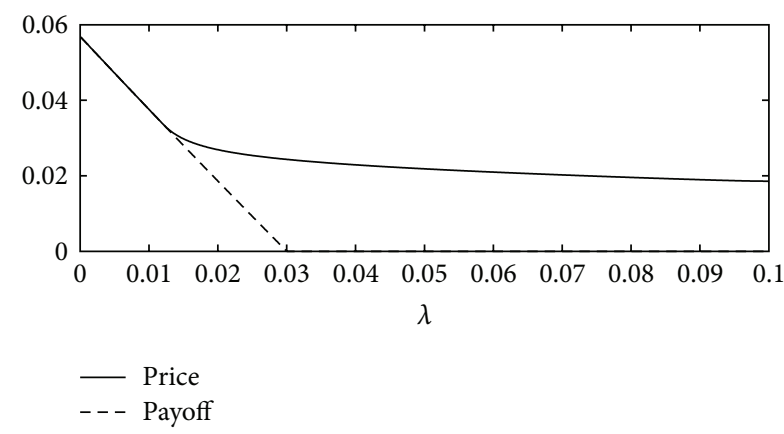

(a)

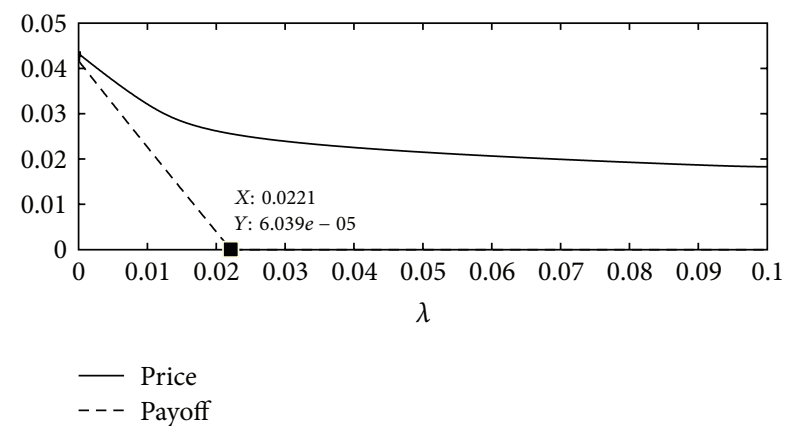

(b)

FIgURE 9: The price of the prepayment option $P_{\Lambda^{*}}(\lambda)$ (solid line) and the payoff $\chi(\lambda)$ (dashed line) as function of the intensity $\lambda$. (a) regime $X=e_{1}$; (b) regime $X=e_{2}$. Two regions appear: the continuation region $\lambda>\Lambda_{1}^{*}$ and the exercise region $\lambda \leq \Lambda_{1}^{*}$. For the second regime there is no exercise region.

option cost is not negligible and should be taken into account in the asset liability management of the bank. Moreover it is seen that when liquidity parameters are very different (i.e., when a liquidity crisis occur) in the high liquidity cost regime, the exercise domain may entirely disappear, meaning that it is not optimal for the borrower to prepay during such a liquidity crisis.

\section{References}

[1] D. Cossin and H. A. Lu, Pricing Prepayment Option in C \& I Loans at Origination, University of Lausanne, School of Economics and Business Administration (HEC-Lausanne).

[2] G. G. Kaufman, T. H. Krueger, and W. C. Hunter, The Asian Financial Crisis: Origins, Implications and Solutions, Springer, 1999.

[3] B. Henry and J. Nixon, "The crisis in Russia: some initial observations," Economic Outlook, vol. 23, no. 1, pp. 22-29, 1998.

[4] J. B. Hillard, J. E. Kau, and V. C. Slawson, "Valuing prepayment and default in a fixed-rate mortgage: a bivariate binomial options pricing technique," Real Estate Economic, vol. 26, no. 3, pp. 431-468, 1998.

[5] Y. Chen, M. Connolly, W. Tang, and T. Su, "The value of mortgage prepayment and default options," Journal of Futures Markets, vol. 29, pp. 840-861, 2009.

[6] E. S. Schwartz and W. N. Torous, "Mortgage prepayment and default decisions: a poisson regression approach," American 
Real Estate and Urban Economics Association, vol. 21, no. 4, pp. 431-449, 1993.

[7] X. Guo and Q. Zhang, "Closed-form solutions for perpetual American put options with regime switching," SIAM Journal on Applied Mathematics, vol. 64, no. 6, pp. 2034-2049, 2004.

[8] X. Guo, "An explicit solution to an optimal stopping problem with regime switching," Journal of Applied Probability, vol. 38, no. 2, pp. 464-481, 2001.

[9] Y. Xu and Y. Wu, "Perpetual American maximum options with Markov-modulated dynamics," Lithuanian Mathematical Journal, vol. 51, no. 1, pp. 106-119, 2011.

[10] R. S. Mamon and M. R. Rodrigo, "Explicit solutions to European options in a regime-switching economy," Operations Research Letters, vol. 33, no. 6, pp. 581-586, 2005.

[11] J. Buffington and R. J. Elliott, "American options with regime switching," International Journal of Theoretical and Applied Finance, vol. 5, pp. 497-514, 2009.

[12] A. Jobert and L. C. G. Rogers, "Option pricing with Markovmodulated dynamics," SIAM Journal on Control and Optimization, vol. 44, no. 6, pp. 2063-2078, 2006.

[13] D. D. Yao, Q. Zhang, and X. Y. Zhou, "A regime-switching model for European options," in Stochastic Processes, Optimization, and Control Theory: Applications in Financial Engineering, \& Management Science2006, H. Yan, G. Yin, and Q. Zhang, Eds., vol. 94 of International Series in Operations ResearchQueueing Networks, and Manufacturing Systems, pp. 281-300, Springer.

[14] T. K. Siu, H. Yang, and J. W. Lau, "Pricing currency options under two-factor Markov-modulated stochastic volatility models," Insurance: Mathematics and Economics, vol. 43, no. 3, pp. 295-2302, 2008.

[15] R. J. Elliott and T. K. Siu, "On Markov-modulated exponentialaffine bond price formulae," Applied Mathematical Finance, vol. 16, no. 1, pp. 1-15, 2009.

[16] N. Zhou and R. Mamon, "An accessible implementation of interest rate models with markov-switching," Expert Systems With Applications, vol. 39, no. 5, pp. 4679-4689, 2012.

[17] Y. Huang, P. A. Forsyth, and G. Labahn, "Methods for pricing American options under regime switching," SIAM Journal on Scientific Computing, vol. 33, no. 5, pp. 2144-2168, 2011.

[18] P. Jaillet, D. Lamberton, and B. Lapeyre, "Variational inequalities and the pricing of American options," Acta Applicandae Mathematicae, vol. 21, no. 3, pp. 263-289, 1990.

[19] J. C. Cox, J. E. Ingersoll, and S. A. Ross, "A theory of the term structure of interest rates," Econometrica, vol. 53, no. 2, pp. 385407, 1985.

[20] A. Aurélien, "On the discretization schemes for the CIR, (and Bessel squared) processes," Monte Carlo Methods and Applications, vol. 11, no. 4, pp. 355-384, 2005.

[21] D. Lamberton and B. Lapeyre, Introduction To sTochastic Calculus Applied To Finance, Chapman \& Hall/ CRC, 2nd edition, 2008.

[22] D. Lando, Credit Risk Modeling, Princeton University Press, Princeton, NJ, USA, 2004.

[23] B. Øksendal, Stochastic Differential Equations, Universitext, Springer, Berlin, Germany, 6 edition, 2007.

[24] M. Abramowitz and I. A. Stegun, Handbook of Mathematical Functions With Formulas, Graphs, and Mathematical Tables, Dover, New York, NY, USA, 9th-10th edition, 1964.

[25] M. Musiela and M. Rutkowski, Martingale Methods in Financial Modelling, Springer, 2005.
[26] R. Myneni, “The pricing of the American option," The Annals of Applied Probability, vol. 2, no. 2, pp. 1-23, 1992.

[27] A. Bensoussan, "On the theory of option pricing," Acta Applicandae Mathematicae, vol. 2, no. 2, pp. 139-158, 1984.

[28] M. Chesney, R. J. Elliott, and R. Gibson, "Analytical solutions for the pricing of American bond and yield options," Mathematical Finance, vol. 3, pp. 277-294, 1993. 


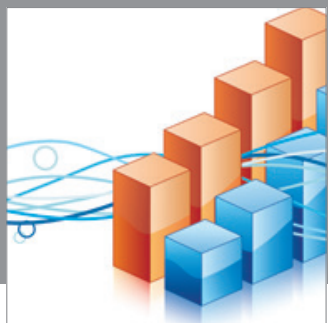

Advances in

Operations Research

mansans

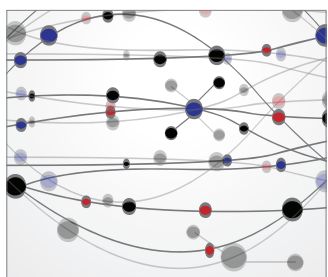

The Scientific World Journal
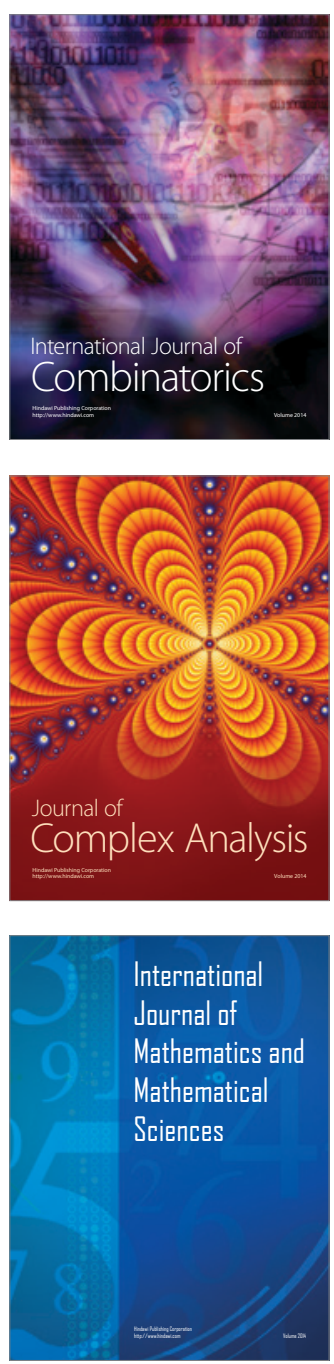
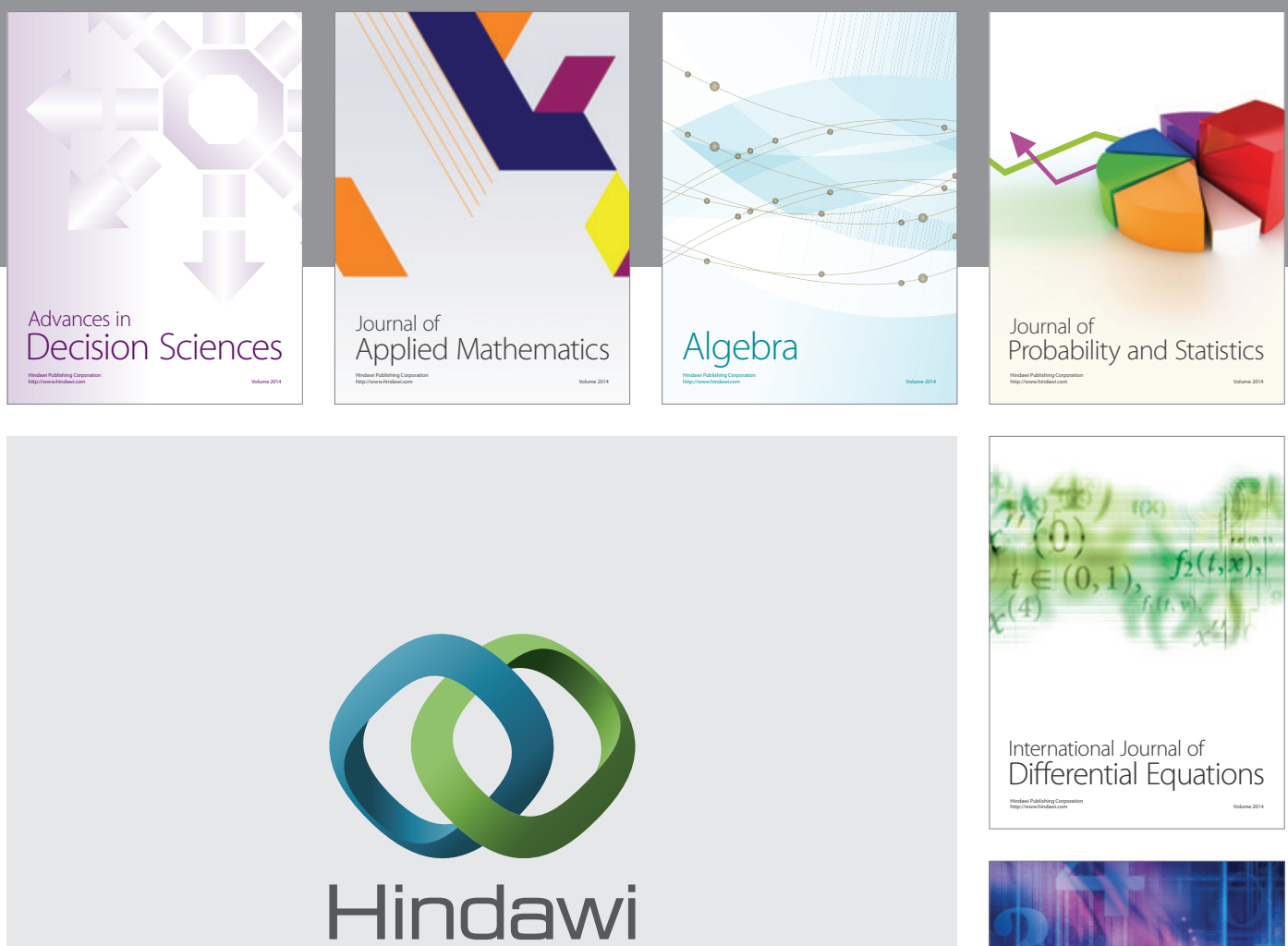

Submit your manuscripts at http://www.hindawi.com
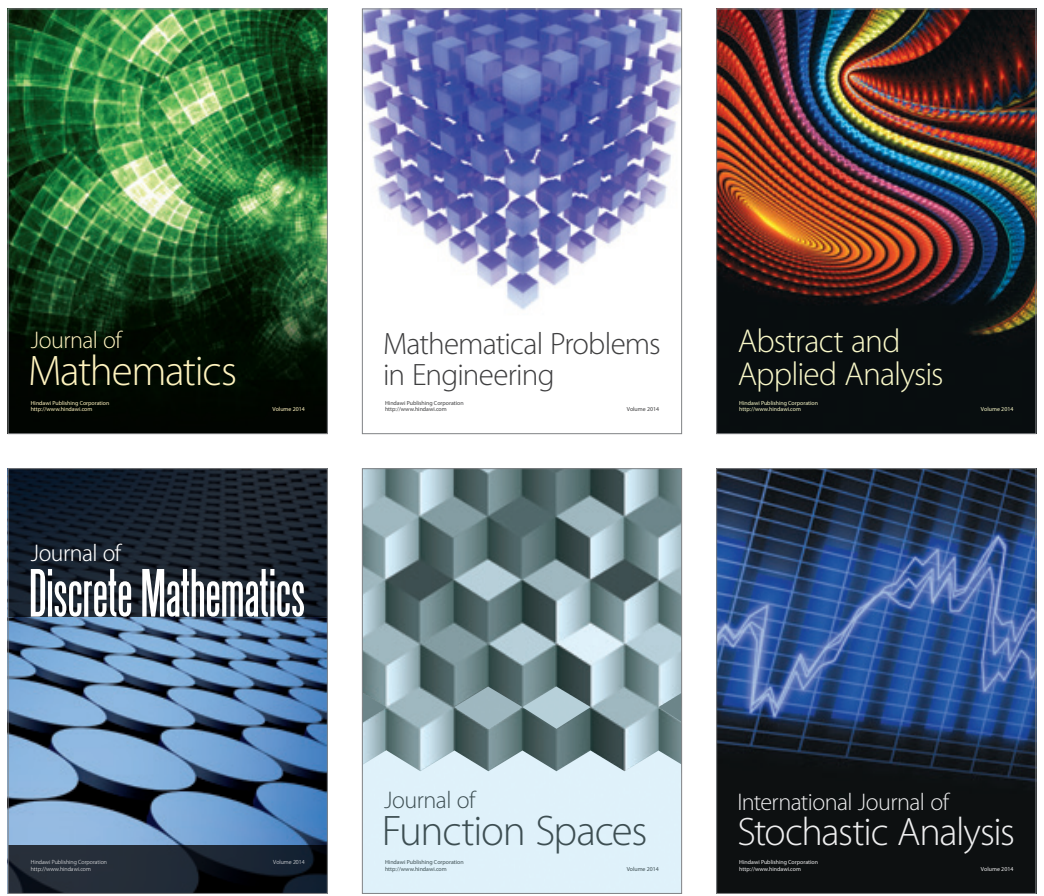

Journal of

Function Spaces

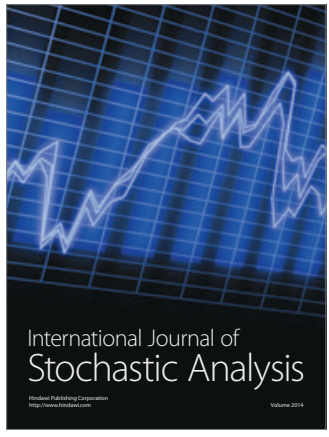

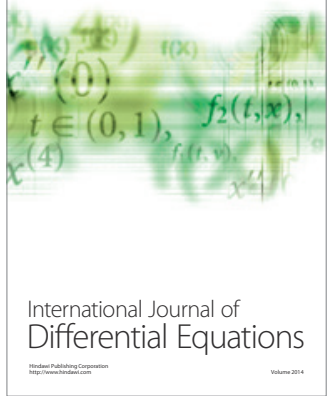
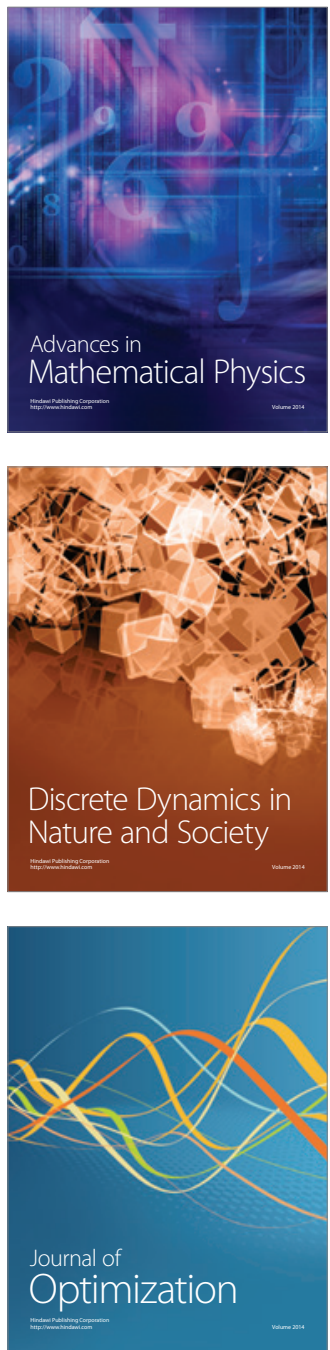